\title{
Early Repolarization Found on Routine Electrocardiograms: Risk and Management
}

Fahed Darmoch, MD, ${ }^{1}$ Toufik Haddad, MD, ${ }^{2}$ Amjad Kabbash, MD, ${ }^{2}$ Hirad Yarmohammadi, MD, ${ }^{3}$ Yasser AI-Khadra, MD, ${ }^{1}$ M. Chadi Alraies, MD $^{4}$

\footnotetext{
${ }^{1}$ Department of Internal Medicine, The Cleveland Clinic, Cleveland, $\mathrm{OH}^{2}$ Department of Cardiology, Creighton University School of Medicine Omaha, NE ${ }^{3}$ Department of Hospital Medicine, Brigham and Women's Hospital, Boston, MA ${ }^{4}$ Department of Internal Medicine, Aultman Hospital, Canton, $\mathrm{OH}$
}

\section{TO THE EDITOR}

Early repolarization (ER) pattern is a prominent $\mathrm{J}$ point (end-QRS notch or slur) with an elevation $\geq 0.1 \mathrm{mV}$ in two or more contiguous leads (excluding V1-V3) in a 12-lead electrocardiogram (ECG). Furthermore, the QRS duration is required to be $<120 \mathrm{~ms}$ (measured in leads in which $\mathrm{J}$ point elevation is absent) with a symmetric concordant $\mathrm{T}$ wave of large amplitude (Figure). ${ }^{1,2}$ ER pattern is most commonly seen in the left lateral leads and less frequently in the inferior leads. ER syndrome, on the other hand, is diagnosed only in patients who have a history of resuscitated cardiac arrest, history of ventricular fibrillation (VF) or poly- morphic ventricular tachycardia. ${ }^{3} \mathrm{ER}$ pattern mimics the ECG patterns seen in myocardial infarction, pericarditis, ventricular aneurysm, hyperkalemia, or hypothermia. Therefore, practitioners often commit to laboratory and imaging tests, medication administration, and hospital admissions that are unnecessary because of ECG misinterpretation.

\section{PREVALENCE AND MECHANISM}

ER pattern is a common ECG finding that is seen in 1\%$5 \%$ of the general population; the majority of patients with ER pattern are male. ${ }^{4}$ Higher prevalences have been reported in African Americans and young healthy athletes. ${ }^{5}$
A
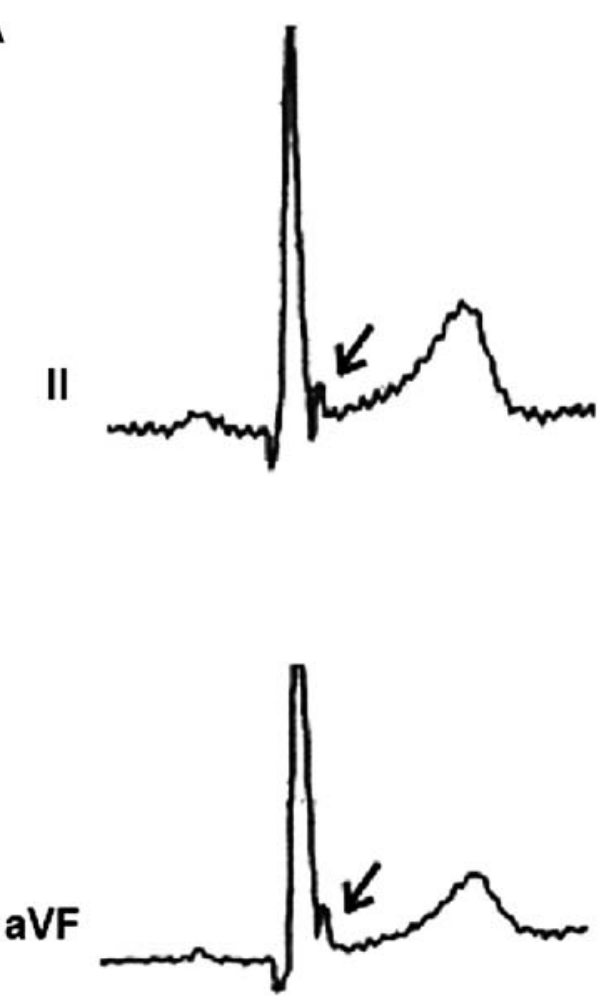

B
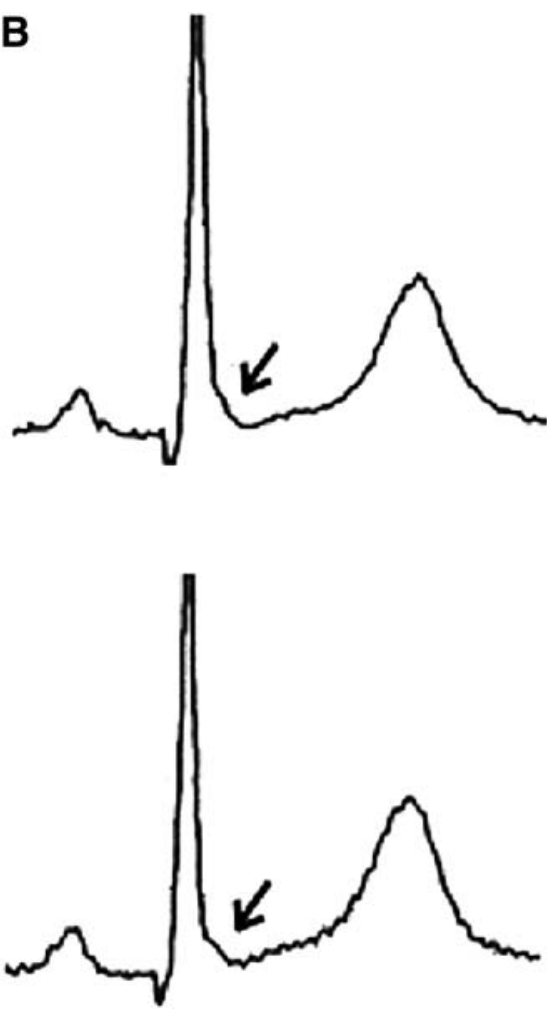

Figure. Early repolarization pattern types seen on routine electrocardiograms. A. Early repolarization with terminal QRS slur on lead II and aVF. B. Early repolarization with terminal QRS notch in lead II and aVF. 
Table. High-Risk Features of Early Repolarization Patterns Seen on Routine Electrocardiograms

J point distribution: Inferior or global distribution across all leads considered high risk compared to lateral distribution that is deemed low risk

J wave amplitude: Amplitude $\geq 0.1 \mathrm{mV}$

ST segment morphology: Horizontal or downsloping ST segment

Coexisting electrical disorder: Conditions such as Brugada syndrome or short QT syndrome

Dynamic J wave: High amplitude and transient augmentation of J wave patterns

Family history of sudden cardiac death: First-degree relative

History of resuscitation from cardiac arrest: Cardiac arrest resulting from documented ventricular fibrillation or polymorphic ventricular tachycardia

While the vast majority of ER is likely sporadic, first-degree relatives of a person with ER pattern appear to have a 2to 3-fold higher likelihood of also having ER pattern. Furthermore, ER pattern was observed in $15 \%$ of cases of idiopathic VF and sudden cardiac death, especially in the 35- to 45year age group. ${ }^{6}$ The mechanism of ER pattern is principally attributable to the lack of synchrony of the isolated regions of the myocardium that repolarize earlier than the rest of the myocardium. This dyssynchrony with increases in repolarizing current or decreases in depolarizing current accelerates repolarization in the myocardium, causing ER pattern. ${ }^{3}$ Although the mechanistic basis of ventricular arrhythmias in patients with ER pattern is still incompletely understood, information about the types of ER is summarized in the Table.

\section{MANAGEMENT}

Because ER pattern is fairly common in the general population, no workup or therapy is recommended in the absence of high-risk features, eg, global or inferior distribu- tion of $\mathrm{J}$ point across all the ECG leads. Furthermore, screening for ER in completely asymptomatic individuals is not appropriate based on the current data. However, for patients with idiopathic VF or resuscitated cardiac arrest without obvious etiology, the correct diagnosis of ER pattern has clinical importance. In these patients, referral to a cardiac electrophysiology specialist is indicated for possible electrophysiology study and possible placement of an implantable cardiac defibrillator for secondary prevention. In summary, emphasis should be placed on the high-risk features listed in the Table to disclose patients with the highest risk for fatal arrhythmias. Finally, young athletes with a benign ECG pattern of ER should not be profiled as high risk, and they do not require specific cardiovascular evaluation as long as they are asymptomatic and have no family history of sudden cardiac death.

\section{REFERENCES}

1. Antzelevitch C, Yan GX, Ackerman MJ, et al. J-wave syndromes expert consensus conference report: emerging concepts and gaps in knowledge. Europace. 2017 Apr 1;19(4):665-694. doi: 10.1093/europace/euw235.

2. Macfarlane PW, Antzelevitch C, Haissaguerre M, et al. The early repolarization pattern: a consensus paper. J Am Coll Cardiol. 2015 Jul 28;66(4):470-477. doi: 10.1016/j.jacc.2015.05.033.

3. Benito B, Guasch E, Rivard L, Nattel S. Clinical and mechanistic issues in early repolarization of normal variants and lethal arrhythmia syndromes. J Am Coll Cardiol. 2010 Oct 5;56 (15):1177-1186. doi: 10.1016/j.jacc.2010.05.037.

4. Wu SH, Lin XX, Cheng YJ, Qiang CC, Zhang J. Early repolarization pattern and risk for arrhythmia death: a metaanalysis. J Am Coll Cardiol. 2013 Feb 12;61(6):645-650. doi: 10.1016/j.jacc.2012.11.023.

5. Aagaard P, Baranowski B, Aziz P, Phelan D. Early repolarization in athletes: a review. Cir Arrhythm Electrophysiol. 2016 Mar;9(3): e003577. doi: 10.1161/CIRCEP.115.003577.

6. Maury $P$, Rollin A. Prevalence of early repolarisation/J wave patterns in the normal population. J Electrocardiol. 2013 SepOct;46(5):411-416. doi: 10.1016/j.jelectrocard.2013.06.014. 